\title{
ETIOLOGÍA NEURÓGENA EN PACIENTES CON DISFUNCIÓN ERECTIL
}

\author{
Consuelo Valles Antuña', J. M. Fernández Gómez 2,3, S. Escaf2,3 y Fernando Fernández- \\ González?.
}

\begin{abstract}
'Servicio de Neurofisiología. Clínica del Hospital Universitario Central de Asturias. Oviedo. España.
2Servicio de Urología I. Hospital Universitario Central de Asturias. Oviedo. España.

${ }^{3}$ Departamento de Cirugía. Facultad de Medicina. Universidad de Oviedo. Asturias. Oviedo. España.
\end{abstract}

\begin{abstract}
Resumen.- OBJETIVO: La disfunción eréctil (DE) es una alteración cuya prevalencia es elevada y aumenta con la edad. Se estima que en España afecta al $18,9 \%$ de los varones de 25 a 70 años. En la mayor parte de los casos es de origen multifactorial y en su patogenia se admite la influencia de enfermedades sistémicas, fármacos de diferentes tipos, factores psicógenos, patologías cardiovasculares, endocrinopatías y alteraciones neurológicas. La disfunción eréctil de causa neurológica puede tener su origen a nivel del Sistema Nervioso Central o Periférico. Entre las posibles causas de disfunción eréctil neurógena de origen central estarían tumores, accidentes cerebrovasculares, encefalitis, Enfermedad de Parkinson, Esclerosis Múltiple y otras
\end{abstract}

Consuelo Valles Antuña Servicio de Neurofisiología Clínica Hospital Universitario Central de Asturias Oviedo. Asturias. (España). consuvall@hotmail.com

Trabajo recibido: 12 de junio 2007 enfermedades desmielinizantes, demencias, degeneración olivopontocerebelosa y epilepsia. Las mielopatías de cualquier etiología, pueden ser dependiendo de su localización o extensión, causas de disfunción eréctil. A nivel periférico pueden ser causa de DE las alteraciones de las vías sensitivas que constituyen el brazo aferente del reflejo espinal de la erección y las de las vías eferentes vegetativas o somáticas que median en la vasodilatación arterial, la relajación del músculo liso cavernoso o la contracción de la musculatura estriada del suelo de la pelvis. La finalidad de este trabajo es revisar detalladamente las causas más relevantes de $D E$ de origen neurógeno, sus mecanismos etiopatogénicos y los abordajes terapéuticos que en la actualidad se consideran más adecuados para cada caso particular.

CONCLUSIÓN: La correcta aproximación diagnóstica al paciente con DE pasa por identificar, en la medida de lo posible, los factores etiopatogénicos implicados su origen. En este sentido, la detección e identificación, de la posible presencia del factor de riesgo neurógeno, contibuirá a un mejor entendimiento de sus mecanismos fisiopatológicos y con ello a una aproximación diagnóstica, pronóstica y terapéutica más adecuada especialmete en aquellos pacientes refractarios a la terapia de primera línea.

Palabras clave: Disfunción eréctil. Etiopatogenia. Enfermedad de Parkinson. Epilepsia. Esclerosis múltiple. Mielopatía. Neuropatía. Polineuropatía. Diagnóstico. Neuromodulación sacra.

Summary.- OBJECTIVES: Erectile dysfunction (ED) is a disorder with a high prevalence that increases with age. It is estimated that $18.9 \%$ of men's between 25 and 70 years suffer it in Spain. Most cases have a multifactorial origin and it is admitted the influence on 
its pathogenesis of systemic diseases, different kind of drugs, psychogenic factors, cardiovascular, endocrinological and neurological diseases. Neurologic cause erectile dysfunction may have its origin in the central or peripheral nervous system. Among possible process of neurogenic erectile dysfunction of central origin would be tumors, cerebral vascular accidents, encephalitis, Parkinson disease, multiple sclerosis and other demyelinization diseases, dementias, olivopontocerebellar degeneration and epilepsy. Myelopathies of any etiology may be, depending on their localization and extension, cause of erectile dysfunction. At the peripheral level, disorders of the sensitive tracts constituting the afferent limb of the erection spinal reflex, and the efferent vegetative or somatic tracts mediating arterial vasodilatation, cavernous smooth muscle relaxation or pelvic floor striated muscle contraction. The aim of this work is to review in detail the most relevant causes of neurogenic erectile dysfunction, their etiopathogenic mechanisms and therapeutic approaches currently considered more adequate for each particular case.

CONCLUSIONS: The correct diagnostic approach to patients with erectile dysfunction passes through identification, if possible, of the etiopathogenic factors implied. Regarding this, detection and identification of a possible neurogenic risk factor will contribute to a better understanding of the physiopathologic mechanisms, and more adequate diagnostic, prognostic and therapeutic approaches, mainly in those patients refractory to first line therapy.

Keywords: Erectile dysfunction. Etiopathogenesis. Parkinson's disease. Epilepsy. Multiple sclerosis. Myelopathy. Neuropathy. Polyneuropathy. Diagnosis. Sacral neuromodulation.

\section{INTRODUCCIÓN}

La disfunción eréctil (DE) es una alteración de alta prevalencia con importantes consecuencias interpersonales y psicosociales. Se estima que en España afecta al 18,9\% de los varones de 25 a 75 años (1). Estudios epidemiológicos a gran escala han demostrado su mayor incidencia en relación con la edad avanzada y con la presencia de otras patologías asociadas (2).

En la actualidad se admite una etiología es multifactorial y por lo general, de origen orgánico. En algunos casos es síntoma centinela de alteraciones cardiovasculares $(3,4)$, enfermedades neurológicas como la atrofia multisitémica (5) o de otros tipos de patologías.
Desde el punto de vista neurológico, cualquier alteración de las vías o centros nerviosos implicados en la erección puede dar origen a DE (Figura 1).

A nivel central incidirían patologías tan frecuentes como accidentes cerebrovasculares y otras mucho mas raras como la degeneración olivopontocerebelosa o el Síndrome de Shy-Drager (6).

Las mielopatías de cualquier etiología, pueden ser dependiendo de su localización o extensión, causas de disfunción eréctil (7).

Sobre el Sistema Nervioso Periférico (SNP) inciden las alteraciones de las vías sensitivas que constituyen el brazo aferente del arco reflejo espinal de la erección y la afectación de las vías eferentes, vegetativas o somáticas, que median la respuesta sexual. Las neuropatías periféricas puden ser locales, como las causadas por traumatismos o cirugía pélvica, y generalizadas como las que ocurren en las polineuropatías tóxicas, metabólicas, infecciosas o congénitas.

Aunque no existen estudios epidemiológicos recientes sobre la prevalencia de la DE neurógena, se estima que es responsable de entre 10 y el $19 \%$ de los casos de DE (8). No obstante, la atención que se le ha prestado clasicamente al factor de riesgo neurógeno ha sido escasa e incluso revisiones recientes, como la publicada por Beutel, en 2006 (9) no se incluye como tal.

\section{CONTROL NEURAL DE LA ERECCIÓN}

La respuesta eréctil depende de una compleja serie de eventos vasculares y neurales que llevan a los vasos y al tejido eréctil del pene desde una situación de contracción y mínima perfusión a una de relajación e ingurgitación.

Los estímulos desencadenantes de la erección pueden ser de tipo psicógeno o sensorial. Los primeros son generados en los centros nerviosos superiores y pueden ser de naturaleza visual, olfativa, táctil o compleja (fantasías, recuerdos, etc). Los estímulos sensitivos locales, desencadenantes de las erecciones reflejas, proceden de los genitales y son conducidos al centro reflejo espinal, situado en la médula sacra, por el nervio pudendo.

La médula espinal contiene los tres grupos de neuronas (simpátíco, parasimpático y somático) ligados anatómicamente con el pene y funcionalmente con la erección. La inervación de los músculos estriados del suelo de la pelvis depende de las 
neuronas somáticas situadas en el Nucleo de Onuff y la contracción de estos músculos induce en el pene erecto un incremento importante de la rigidez y de la presión intracavernosa (10) y su actividad rítmica participa en la expulsión del semen durante la eyaculación.

La ME es una estructura clave sobre la que confluye la información de tipo excitatorio o inhibitorio procedente de la periferia y de los núcleos supraespinales implicados en la erección; es también responsable de coordinar la erección con las otras fases de la respuesta sexual y de inhibir funciones pélvicas como la micción o la defecación.

A nivel supraespinal, diversas estructuras como el área preóptica medial, la porción parvocelular del núcleo paraventricular del hipotálamo, el nucleo paragigantocelular del mesencéfalo y el núcleo medial de la amígdala, intervienen en el control de la erección mediante una función efectora directa sobre la médula o mediante la integración de las sensa- ciones con cogniciones, fantasías, memorias y otros inputs. Es posible que diferentes áreas del encéfalo contribuyan al desarrollo de la respuesta sexual en diferentes contextos, algunos de ellos sin contenido sexual relevante (11). Así, la respuesta sexual podría reflejar, en cada contexto, la contribución de una combinación única de varios núcleos encefálicos y en el mismo sentido, cada núcleo podría contribuir a la génesis de la erección en diferentes contextos. Por otra parte, la actividad de cada núcleo podría depender de la información que recibe de la periferia y de otros núcleos centrales y, por supuesto, de su ambiente hormonal.

\section{D.E. NEURÓGENA DE ORIGEN CENTRAL}

a. Esclerosis Múltiple. (EM) Es una alteración de curso variable en su distribución espacial y temporal, caracterizada por las presencia de múltiples áreas de inflamación y desmielinización en el SNC. Sin patrón de presentación o curso clínico definidos, la

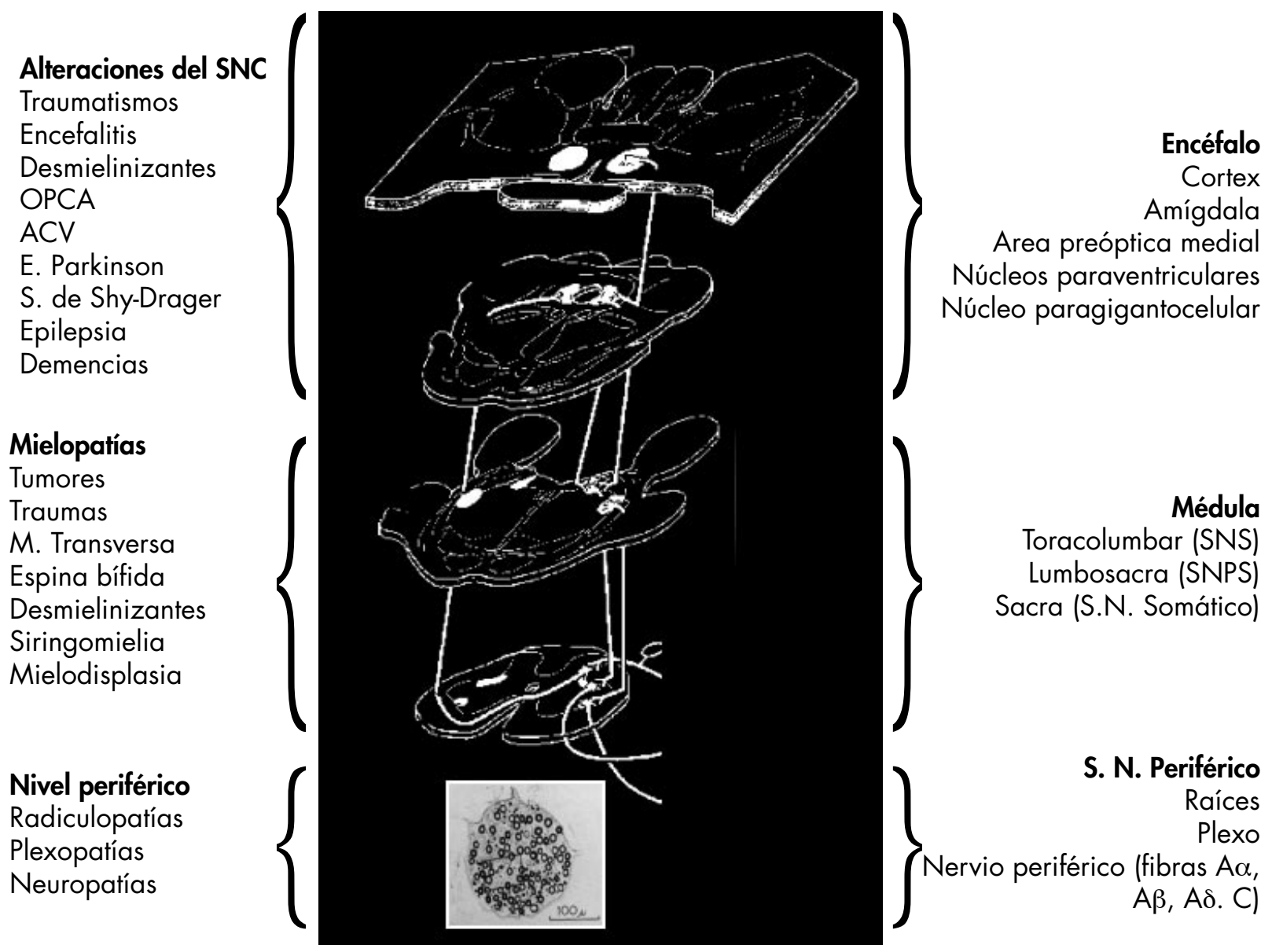

FIGURA 1. Causas neurológicas de disfunción eréctil, según nivel de afectación. 
sintomatología más común incluye temblor, neuritis óptica, diplopia, disartria, discinesia y síntomas genitourinarios.

La relación entre EM y la disfunción sexual fue sugerida incialmente por VAS en 1969 (12). Es un problema que repercute negativamente sobre la calidad de vida y se presenta incluso en pacientes sin discapacidad severa (13). Afecta al $91 \%$ de los hombres (14), y el síntoma más frecuente es la DE $(63,2 \%)$, seguida de las alteraciones de la eyaculación o el orgasmo $(50 \%)$ y la disminución de la libido $(39,5 \%) \quad(15,16)$. Al principio el déficit es parcial, preservándose las erecciones nocturnas y matutinas (17), pero al avanzar el déficit neurológico puede haber un fallo total de la erección (18).

Factores con impacto negativo sobre la función sexual de estos pacientes serían el sexo masculino, la edad, el bajo nivel de educación, el mayor tiempo de evolución, el curso primariamente progresivo, la presencia de depresión o ansiedad y la disfunción urinaria o esfinteriana asociadas (16). Influyen también la función cognitiva, el nivel de autonomía, el tiempo de evolución de la enfermedad y la atrofia del parénquima del pons (19). No debe olvidarse la posible intervención de aspectos como la fatiga, las alteraciones urinarias o los efectos secundarios de los fármacos empleados en el tratamiento de la enfermedad (20).

b. Enfermedad de Parkinson (EP). La enfermedad de Parkinson es un trastorno neurodegenerativo caracterizado por síntomas motores y por signos como temblor, rigidez y bradicinesia que se manifiestan cuando se ha perdido sobre el $70 \%$ de las neuronas dopaminergicas de la sustancia nigra.

Con la progresión de la enfermedad el proceso neurodegenerativo se extiende hasta afectar a las regiones mesolímbica y mesocortical y a las áreas autonómicas del Sistema Nervioso Central (SNC). Es entonces cuando los pacientes desarrollan síntomas no motores incluyendo la DE (21).

La frecuente asociación con DE (22) no es sorprendente, dada la importancia de las vías dopaminérgicas en la libido y en la erección. Por otro lado, la disautonomía en la EP ha sido descrita extensamente. Los síntomas son variables y afectan al $80 \%$ de los pacientes (23).

La DE en pacientes con EP ha sido relacionada con la gravedad de la dolencia, el tiempo de evolución, los síntomas motores, la depresión, la alteración de la imagen corporal, la falta de autonomía para las actividades de la vida diaria, la disfunción vegetativa y los efectos secundarios de la medicación antiparkinsoniana $(24,25)$. Los antecedentes de disfución sexual premórbida influyen sobre la libido de los pacientes durante la enfermedad (26).

La hipersexualidad, con o sin manía asociada, ha sido descrita en pacientes tratados con estimulación cerebral profunda del Núcleo Subtalámico (27).

El tratamiento de elección en estos estos pacientes son los inhibidores de la 5PDE y la PGE 1 . Otras posibles vías de actuación terapeútica sobre la ED asociada a EP podrían orientarse a la actuación sobre el SNC lagonistas dopaminérgicos, estimulación cerebral profunda y ligandos de neuroinmunofilinas) o a nivel intracavernoso (inhibidores de la 5PDE y PGE I) (28).

\section{c. Atrofia multisistémica (Síndrome de Shy Drager).}

Es una enfermedad neurodegenerativa progresiva caracterizada por parkinsonismo y disfunciones cerebelosa, autonómica, urinaria y/o piramidal. La supervivencia media, tras el primer síntoma, de 9,3 años (29).

En cerca del $40 \%$ de los casos la DE es el primer síntoma y precede en años a otros síntomas (30) como las alteraciones urinarias y la hipotensión ortostática (31). Se ha propuesto $(30,31)$ la alteración de las vías dopaminérgicas en el SNC como posible mecanismo fisiopatológico de la DE. La presentación combinada de disfunción eréctil y vesical ha de alertar al urólogo sobre la posibidad de atrofia multisistémica subyacente (31). El hecho de que sildenafil pueda exacerbar los síntomas de hipotensión ortostática ha de tenerse en cuenta en estos pacientes (32).

d. Epilepsia. Los pacientes epilépticos tienen un riesgo de desarrollar disfunción eréctil del 57\%, (33) sensiblemente mayor al detectado en la población general (34).

La prevalencia varía con la gravedad de la enfermedad y oscila del $3 \%$ en pacientes ambulatorios (35) al 58\% en pacientes con epilepsia grave, subsidiaria de tratamiento quirúrgico (36). Factores como el stress o un mayor número de crisis favorecen el desarrollo de DE (36-38).

La incidencia es mayor en los pacientes con epilepsia del lóbulo temporal que en aquellos con epilepsia primaria generalizada $(39,40)$.

Desde el punto de vista fisiopatológico, la disfunción sexual en los pacientes epilépticos parece ser multifactorial. Entre los mecanismos propuestos se encuentran: 
a) cambios en la función vegetativa inducidos por las crisis y/o por las descargas epileptiformes;

b) alteraciones de la función neuroendocrinas inducidas, directamente o mediante un efecto neuromodulador, por las descargas epileptiformes $(41,42)$;

c) los fármacos antiepilépticos que alteran, por varios mecanismos, las concentraciones séricas de hormonas sexuales y/o el feedback regulador del eje hipotálamo-hipófiso-gonadal (43) y (d) la influencia de factores psicosociales.

e. Accidentes cerebrovasculares (ACV). La disfunción sexual y la insatisfacción con la vida sexual son comunes en los pacientes con accidentes cerebrovasculares y en sus parejas. La edad, el sexo, la localización del ACV, la extensión de las lesiones y la asociación con hipertensión arterial y/o diabetes se correlacionan con la disminución a medio y largo plazo de la actividad sexual (44). Muchos de los agentes antihipertensivos, con los que a menudo se trata a los pacientes con $A C V$, inhiben o dificultan la erección al disminuir la presión en las arterias cavernosas y algunos, como los $\beta$-bloqueantes, disminuyen la libido.

f. Demencia de Alzheimer. La disfunción eréctil ha sido descrita en esta enfermedad (41) en la que uno de los síntomas afectivos más frecuentes son las alteraciones de la conducta sexual (46). Las características generales los pacientes, la severidad de la enfermedad, la sintomatología depresiva, la edad, el déficit cognitivo o la relación previa con la pareja no parecen mostrar correlación con las alteraciones de la conducta sexual (47).

g. Traumatismos craneoencefálicos (TCE). La alta tasa de prevalencia de DE descrita en pacientes con antecedente de TCE (36 al 54\%) y se debe no sólo al daño cerebral postraumático sino tambien a los efectos no cerebrales de la injuria como las alteraciones de la conducta o del humor, la disfunción musculoesquelética, etc. El pronóstico debería venir basicamente determinado por la extensión y localización de la lesión, pero se ha detectado influencia de los aspectos psicosociales y afectivos, siendo la presencia de depresión el predictor más sensible en cuanto a la evolución de disfunción sexual (48).

\section{DIFUNCIÓN ERÉCTIL DE ORIGEN MEDULAR.}

El impacto de las lesiones medulares en la función sexual dependerá de su localización y extensión (49). Los pacientes con lesiones altas de la ME (torácicas) mantienen las erecciones reflejas, mientras que los que tienen lesiones bajas pueden experimentar erecciones psicógenas. Sin embargo, aunque la tumescencia peneana sea posible, no siempre se obtiene una erección de calidad o duración suficientes para el coito.

La disfunción eréctil en los lesionados medulares se atribuye por norma general a causas neurógenas, ya que la concurrencia de enfermedad vascular significativa o de neuropatía periférica de otro origen suele ser baja. La presencia de alteraciones vesicales y esfinterianas asociadas o el uso frecuente en estos pacientes de fármacos como baclofén, antidepresivos o antihipertensivos ha de ser tenido en cuenta. La incidencia de disfunción eréctil de causa psicógena puede llegar a superar el 10\% (50).

Respecto al tratamiento, se recomienda un abordaje multidisciplinar, teniendo en cuenta la edad y el perfil psicológico del lesionado y de su pareja. Determinados riesgos y complicaciones, como equimosis, priapismo y disreflexia autonómica, pueden ser más frecuentes en la población con lesión medular que en aquellos pacientes con disfunción debida a causa vascular. Así, los lesionados medulares suelen responder en demasía a las inyecciones intracavernosas de agentes vasodilatadores, debido a la hipersensibilidad de los receptores y a la falta de factores comórbidos, en una población, por lo general, joven.

Otras terapias poco empleadas, como la MUSE (Medicated Intrauretral System for Erection) generalmente con PGE suelen requerir también ajuste de dosis con el fin de prevenir posibles complicaciones como el síncope y la hipotensión ortostática.

\section{DIFUNCIÓN ERECTIL DE ORIGEN PERIFÉRICO}

Las alteraciónes de raíces sacras, del plexo pudendo o de sus ramas, al igual que la afectación más o menos generalizada de los troncos nerviosos periféricos, pueden dar lugar a DE.

a. Polineuropatía diabética. La DE es la forma más común de disfunción sexual en varones diabéticos, con una incidencia estimada del 35 al $75 \%$ (51). Se considera marcador de desarrollo de enfermedad vascular generalizada y presagio de un posible fallo cardiovascular $(52,53)$. Se acepta que la DE en Diabetes Mellitus (DM) resulta de una combinación de alteraciones que afecta a casi cada una de las etapas de la erección del pene. Esto incluye fallos en la transmisión de señales nerviosas, alteración de células endoteliales con disfución en la liberación de NO por las mismas, disminución del flujo aferente 
arterial debido a enfermedad vascular periférica y defecto en la relajación del músculo liso cavernoso por glicosilación de fibras elásticas (54).

La incidencia de neuropatía diabética se relaciona con el control de la glucemia (55), y se estima que un $70 \%$ de los pacientes mal controlados la desarrollarán en los 25 años de evolución de la enfermedad. Desafortunadamente, cuando el paciente diabético acude a consulta por disfunción eréctil, la neuropatía suele ser la clave en la fisiopatología de la enfermedad.

Un dato a tener en cuenta en el tratamiento de los pacientes diabéticos con ED es que el óxido nítrico (NO), neurotransmisor clave en la erección, puede ser un agente activo en la muerte de los nervios no adrenérgicos-no colinérgicos y que este efecto sería exacerbado por la pérdida de equivalentes reductores, que amplifican la injuria oxidativa $(56,57)$.

b. Neuropatía Alcohólica. Es la más frecuente de las polineuropatías tóxicas en nuestro medio. Su prevalencia es difícil del determinar. En un estudio realizado en 1995 entre 107 pacientes con alcoholismo crónico, el $24.3 \%$ cumplían criterios de neuropatía autonómica y el $32 \%$ cumplían criterios neurofisiológicos de polineuropatía (58).

En la fisiopatología de la neuropatía asociada a alcoholismo crónico se admite que influyen tanto factores de déficit nutricional (59) como el efecto tóxico primario del alcohol sobre el sistema nervioso periférico. Los síntomas suelen ser preferentemente sensitivos, de evolución lentamente progresiva, con afectación predominante de la sensibilidad superficial y sobre todo de la nocicepción, y con disestesias de tipo urente o quemante. En el déficit de tiamina suele darse una afectación motora de evolución aguda y progresiva con disminución de la sensibilidad superficial y profunda. La biopsia del nervio sural suele ser también diferente, con afectación predominante de fibras finas en la al neuropatía alcohólica y axonal de fibras gruesas en el deficit de tiamina. La neuropatía autonómica es, con frecuencia, asintomática en estos pacientes, siendo la DE el síntoma mas frecuente entre los pacientes que presentan clínica (60).

c. Síndrome de Charcot-Marie-Tooth. Polineuropatía poco frecuente que incluye una serie de enfermedades genéticas heterogeneas, caracterizadas por desmielinización, hipertrofia neural (onion bulb formation) y alteración de los reflejos osteotendinosos (61). Se ha descrito, a diferencia de en otras polineuropatías genéticas (62), clínica de DE en estos pacientes. d. Polineuropatía amiloide familiar. Grupo que incluye más de 40 neuropatías de herencia autosómica dominante. En su evolución, se acompaña de polineuropatía sensitivomotora y autonómica progresivas. Uno de los síntomas iniciales mas común es la disfunción eréctil (63), siendo también frecuentes las alteraciones de la micción. El transplante hepático, una de las terapias mas utilizadas en estos pacientes, no mejora la disfunción sexual. Se ha descrito un caso, del tipo japonés, en el que la función sexual mejoró con $25 \mathrm{mg}$ de sildenafil, tratamiento que permitió obtener una rigidez completa (64).

e. Cirugía pélvica. La DE es común tras la prostatectomía radical y afecta del 14 al $84 \%$ de pacientes sometidos a cirugía del cancer de próstata localizado. Su etilogía parece ser multifactorial, siendo fundamental el papel del componente neurógeno. La cirugía selectiva, con preservación bilateral del paquete vasculo-nervioso pudendo, mejora importantemente el pronóstico. De esta forma, se encontró clara correlación entre el número de bucles neurovasculares preservados y la función postquirúrgica (65). La edad influye en el pronóstico, de forma que los pacientes más jóvenes necesitarían conservar un menor número de bucles neurovasculares para mantener una adecuada función $(66,67)$. Otro factor que influye en el mantenimiento postquirúrgico de la función eréctil es la función previa a la cirugía $(68,69)$.

Otros procedimiento quirúrgicos potencialmente responsables de DE serían la cistectomía radical y la excisión mesorectal total, utilizados respectivamente para el tratamiento del cáncer de vejiga y recto. Tambien en estos pacientes, los procedimientos de cirugía selectiva favorecen una mejor función sexual posterior.

En cuanto al tratamiento, los programas de rehabilitación asistida farmacologicamente, con inhibidores de la 5-PDE o con inyección intracavernosa de PG son, en el momento actual, un standard en evolución (70).

Los inhibidores de la 5-PDE pueden ser inutiles en aquellos pacientes en los que la inervación autonómica pélvica haya sido dañada bilateralmente, pues su eficacia depende del mantenimiento de una integridad neural suficiente como para suplir la cantidad mínima necesaria de Oxido Nítrico. La neuromodulación y la neuroestimulación se han propuesto como aproximación útil para mejorar la función eréctil tras la cirugía pélvica (71). 


\section{CONCLUSIÓN}

El diagnóstico y tratamiento de la DE proporcionan una excelente oportunidad para mejorar la calidad de vida del paciente que la padece. Dado que se trata de una patología de etiología multifactorial en la que los factores de riesgo conocidos pueden no ser equivalentes a las causas subyacentes reales, una correcto abordaje diagnóstico pasa, necesariamente, por identificar, en la medida de lo posible, los factores etiopatogénicos implicados su origen.

El conocimiento detallado de las posibles causas de afectación neurógena en DE puede contribuir a un mejor entendimiento de sus mecanismos fisiopatológicos y con ello a una aproximación diagnóstica, pronóstica y terapéutica más adecuada a cada caso particular y especialmete en aquellos pacientes refractarios a la terapia de primera línea. Por otro lado, la indicación responsable de terapias emergentes, como la neuromodulación ha de pasar necesariamente por la identificación clínica y neurofisiológica de una posible alteración neurógena.

\section{BIBLIOGRAFÍA y LECTURAS RECOMENDADAS ( ${ }^{*}$ lectura de interés $y^{* *}$ lectura fundamental)}

*1. MARTIN-MORALES, A.; SANCHEZ-CRUZ, J.J.; SAENZ DE TEJADA, I. y cols.: "Prevalence and independent risk factors for erectile dysfunction in Spain: Results of the epidemiologia de la disfuncion erectil masculina study". J. Urol., 166: $569,2001$.

2. ROSEN, R.C.; WING, R.; SCHNEIDER, S. y cols.: "Epidemiology of erectile dysfunction: The role of medical comorbidities and lifestyle factors". Urol. Clin. North Am., 32: 403, 2005.

3. SADOVSKY, R.; MINER, M.: "Erectile dysfunction is a signal of risk for cardiovascular disease: A primary care view". Prim. Care., 32: 977, 2005.

4. BURNETT, A.L.: "Erectile dysfunction”.J. Urol., 175: 25, 2006.

5. KIRCHHOF, K.; APOSTOLIDIS, A.N.; MATHIAS, C.J. y cols.: "Erectile and urinary dysfunction may be the presenting features in patients with multiple system atrophy: A retrospective study". Int. J. Impot. Res., 15: 293, 2003.

6. LUE, T.F.: "Physiology of penile erectión and pathophysiology of erectile dysfunction and priapism" En WALSH, P.C.; RETIK, A.B.; VAUGHAN, E.D. y cols. (eds): CAMPBELL'S UROLOGY, 8th ed. Philadelphia Saunders, Cap. 45, 2002.
*7. STEERS, W.D.: "Neural pathways and central sites involved in penile erection: Neuroanatomy and clinical implications". Neurosci. Biobehav. Rev., 24: 507, 2000.

*8. SAENZ DE TEJADA, I.; ANGULO, J.; CELLEK, S. y cols.: "Physiology of erectile function". J. Sex. Med., 1: 254, 2004.

9. BEUTEL, M.E.; WEIDNER, W.; BRAHLER, E.: "Epidemiology of sexual dysfunction in the male population". Andrologia, 38: 115, 2006.

10. SCHMIDT, M.H.; SCHMIDT, H.S.: "The ischiocavernosus and bulbospongiosus muscles in mammalian penile rigidity”. Sleep., 16: 171, 1993.

11. SACHS, B.D.: "Contextual approaches to the physiology and classification of erectile function, erectile dysfunction, and sexual arousal". Neurosci. Biobehav. Rev., 24: 541, 2000.

12. VAS, C.J.: "Sexual impotence and some autonomic disturbances in men with multiple sclerosis". Acta Neurol. Scand., 45: 166, 1969.

13. DEMIRKIRAN, M.; SARICA, Y.; UGUZ, S. y cols.: "Multiple sclerosis patients with and without sexual dysfunction: are there any differences?". Mult. Scler., 12: 209, 2006.

14. LILIUS, H.G.; VALTONEN, E.J.; WIKSTROM, J.: "Sexual problems in patients suffering from multiple sclerosis". Scand. J. Soc. Med., 4: 41, 1976.

15. ZORZON, M.; ZIVADINOV, R.; BOSCO, A. y cols.: "Sexual dysfunction in multiple sclerosis: A case-control study. I. Frequency and comparison of groups". Mult. Scler, 6: 418, 1999.

16. ZIVADINOV, R.; ZORZON, M.; BOSCO, A. y cols.: "Sexual dysfunction in multiple sclerosis: II. Correlation analysis". Mult. Scler., 5: 428, 1999.

17. KIRKEBY, H.J.; POULSEN, E.U.; PETERSEN, T. y cols.: "Erectile dysfunction in multiple sclerosis". Neurology, 38: 1366, 1988.

18. BETTS, C.D.; JONES, S.J.; FOWLER, C.G. y cols.: "Erectile dysfunction in multiple sclerosis. Associated neurological and neurophysiological deficits, and treatment of the condition". Brain, 117: 1303, 1994.

19. ZIVADINOV, R.; ZORZON, M.; LOCATELLI, L. y cols.: "Sexual dysfunction in multiple sclerosis: A MRI, neurophysiological and urodynamic study". Neurol. Sci., 15: 73, 2003.

20. LANDTBLOM, A.M.: "Treatment of erectile dysfunction in multiple sclerosis". Expert. Rev. Neurother., 6: 931, 2006.

21. FEARNLEY, J.M.; LEES, A.J.: "Ageing and Parkinson's disease: Substantia nigra regional selectivity". Brain., 114: 2283, 1991. LUCON, M.; DE SOUZA SANTOS, A.; PINTO, A.S. y cols.: "Assessment of erectile dysfunction in patients with Parkinson's disease". Arq. Neuropsiquiatr., 59: 559, 2001. 
*22. LAMBERT, D.; WATERS, C.H.: "Sexual dysfunction in Parkinson's disease". Clin. Neurosci., 5: 73, 1998

23. JOST, W.H.: "Autonomic dysfunctions in idiopathic Parkinson's disease". J. Neurol., 250: 28, 2003.

24. JIMENEZ-JIMENEZ, F.J.; TALLON-BARRANCO, A.; CABRERA-VALDIVIA, F. y cols.: "Fluctuating penile erection related with levodopa therapy". Neurology, 1: 210, 1999.

25. SINGER, C.; WEINER, W.J.; SANCHEZ-RAMOS, J.R.: "Autonomic dysfunction in men with Parkinson's disease". Eur. Neurol., 32: 134, 1992.

26. BRONNER, G.; ROYTER, V.; KORCZYN, A.D. y cols.: "Sexual dysfunction in Parkinson's disease".J. Sex. Marital. Ther., 30: 95, 2004.

27. ROMITO, L.M.; RAJA, M.; DANIELE, A. y cols.: "Transient mania with hypersexuality after surgery for high frequency stimulation of the subthalamic nucleus in Parkinson's disease". Mov. Disord., 17: 1371, 2002.

*28. PAPATSORIS, A.G.; DELIVELIOTIS, C.; SINGER, C. y cols.: "Erectile dysfunction in Parkinson's disease”. Urology,67: 447, 2006.

29. KLOCKGETHER, T.; LÜDTKE, R.; KRAMER, B. y cols.: "The natural history of degenerative ataxia: A retrospective study in 466 patients". Brain, 121: 589, 1998.

30. BECK, R.O.; BETTS, C.D.; FOWLER, C.J.: "Genitourinary dysfunction in multiple system atrophy: Clinical features and treatment in $62 \mathrm{ca}-$ ses". J. Urol., 151: 1336, 1994.

31. KIRCHHOF, K.; APOSTOLIDIS, A.N.; MATHIAS, C.J. y cols.: "Erectile and urinary dysfunction may be the presenting features in patients with multiple system atrophy: A retrospective study". Int. J. Impot. Res., 15: 293, 2003.

32. HEATON, J.P.: "Central neuropharmacological agents and mechanisms in erectile dysfunction: The role of dopamine". Neurosci. Biobehav. Rev., 24: 561,2000

33. TOONE, B.K.: "Sexual disorders in epilepsy". Pedley, T.A.; Meldrum, B.S. editors. Recent advances in epilepsy, vol. 3. Edinburgh: Churchill Livingstone; pág. 233-59, 1986.

34. FELDMAN, H.A.; GOLDSTEIN, I.; HATZICHRISTOU, D.G. y cols.: "Impotence and its medical and psychosocial correlates: Results of the Massachusetts male aging study". J. Urol., 151: 54, 1994.

35. REMILLARD, G.M.; ANDERMANN, F., TESTA, G.F. y cols.: "Sexual ictal manifestations predominate in women with temporal lobe epilepsy: A finding suggesting sexual dimorphism in the human brain". Neurology, 33: 323, 1983.

36. JENSEN, P.; JENSEN, S.B.; SORENSEN, P.S. y cols.: "Sexual dysfunction in male and female patients with epilepsy: A study of 86 outpatients". Arch. Sex.b Behav., 19: 1, 1990.

37. DUNCAN, S.; BLACKLAW, J.; BEASTALL, G.H. y cols.: "Sexual function in women with epilepsy". Epilepsia, 38: 1074, 1997.

38. DANIELE, A.; AZZONI, A.; BIZZI, A. y cols.: "Sexual behavior and hemispheric laterality of the focus in patients with temporal lobe epilepsy". Biol. Psychiatry, 1: 617, 1997.

39. SHUKLA, G.D.; SRIVASTAVA, O.N.; KATIYAR, B.: "Sexual disturbances in temporal lobe epilepsy: A controlled study". Br. J. Psychiatry., 134: 288, 1979.

40. WAXMAN, S.G.; GESCHWIND, N.: "The interictal behavior syndrome of temporal lobe epilepsy". Arch. Gen. Psychiatry, 32: 1580, 1975.

41. SPARK, R.F.; WILLS, C.A.; ROYAL, H.: "Hypogonadism, hyperprolactinaemia, and temporal lobe epilepsy in hyposexual men". Lancet, 1: 413, 1984.

42. PRITCHARD, P.B.3rd; WANNAMAKER, B.B.; SAGEL, J. y cols.: "Endocrine function following complex partial seizures". Ann. Neurol., 1: 27, 1983.

43. TOONE, B.K.; WHEELER, M.; FENWICK, P.B.: "Sex hormone changes in male epileptics". Clin. Endocrinol. (Oxf), 12: 391, 1980.

44. CHOI-KWON, S.; KIM, J.S.: "Poststroke emotional incontinence and decreased sexual activity". Cerebrovasc. Dis., 13: 31, 2002.

45. ZEISS, A.M.; DAVIES, H.D.; WOOD, M. y cols.: "The incidence and correlates of erectile problems in patients with Alzheimer's disease". Arch. Sex. Behav., 19: 325, 1990.

46. DEROUESNE, C.; PIQUARD, A.; THIBAULT, S. y cols.: "Noncognitive symptoms in Alzheimer's disease. A study of 150 communitydwelling patients using a questionnaire completed by the caregiver". Rev. Neurol. (Paris), 157: $162,2001$.

47. DEROUESNE, C.; GUIGOT, J.; CHERMAT, V. y cols.: "Sexual behavioral changes in Alzheimer disease". Alzheimer Dis. Assoc. Disord., 10: 86, 1996.

48. HIBBARD, M.R.; GORDON, W.A.; FLANAGAN, S. y cols.: "Sexual dysfunction after traumatic brain injury". NeuroRehabilitation, 15: 107, 2000.

49. FISHER, T.L.; LAUD, P.W.; BYFIELD, M.G. y cols.: "Sexual health after spinal cord injury: A longitudinal study". Arch. Phys. Med. Rehabil., 83: 1043, 2002.

*50. RAMOS, A.S.; SAMSO, J.V.: "Specific aspects of erectile dysfunction in spinal cord injury". Int. J. Impot. Res., 16: 42, 2004.

51. McCULLOCH, D.K.; CAMPBELL, I.W.; WU, 
F.C. y cols.: "The prevalence of diabetic impotence". Diabetologia, 18: 279, 1980.

52. VINICK, A.I.; RICHARDSON, D.: "Erectile dysfunction in diabetes". Diabetes Reviews, 6: 16, 1988.

53. VINICK, A.I.; MASER, R.E.; MITCHELL, B.D. y cols.: "Diabetic autonomic neuropathy". Diabetes Care, 26: 5, 2003.

*54. RICHARDSON, D.; VINIK, A.: "Etiology and treatment of erectile function in diabetes mellitus". Current Diabetes Reports, 2: 501, 2002.

55. PIRAT, J.: "Diabetes Mellitus and its degenerative complications: A prospective study of 4440 patientes observed between 1947 and 1973". Diabetes Care, 1: 168, 1978.

56. CELLEK, S.; RODRIGO, J.; LOBOS, E. y cols.: "Selective nitrergic neurodegeneration in diabetes mellitus - a nitric oxide-dependent phenomenon". Br. J. Pharmacol., 128: 1804, 1999.

57. KAHN, A.M.; ALLEN, J.C.; SEIDEL, C.L. y cols.: "Insulin increases NO-stimulated guanylate cyclase activity in cultured VSMC while raising redox potential". Am. J. Physiol. Endocrinol. Metab., 278: 627, 2000.

58. MONFORTE, R.; ESTRUCH, R.; VALLS-SOLE, J. y cols.: "Autonomic and peripheral neuropathies in patients with chronic alcoholism. A doserelated toxic effect of alcohol". Arch. Neurol., 52: 45, 1995.

59. VICTOR, M.: "Nutrition and diseases of the nervous system (including psychiatric disorders)". Prog. Food Nutr. Sci., 1: 145, 1975.

60. RAVAGLIA, S.; MARCHIONI, E.; COSTA, A. y cols.: "Erectile dysfunction as a sentinel symptom of cardiovascular autonomic neuropathy in heavy drinkers". J. Peripher. Nerv. Syst., 9: 209, 2004.

61. KELLER, M.P.; CHANCE, P.F.: "Inherited peripheral neuropathy". Semin. Neurol., 19: 353, 1999.

62. BIRD, T.D.; LIPE, H.P.; CRABTREE, L.D.: "Im- potence associated with the Charcot-Marie-Tooth syndrome". Eur. Neurol., 34: 155, 1994.

63. COELHO, T.: "Familial amyloid polyneuropathy: New developments in genetics and treatment". Curr. Opin. Neurol., 9: 355, 1996.

64. OBAYASHI, K.; ANDO, Y.; TERAZAKI, H. y cols.: "Effect of sildenafil citrate (Viagra) on erectile dysfunction in a patient with familial amyloidotic polyneuropathy ATTR Val30Met". J. Auton. Nerv. Syst., 12: 89, 2000.

*65. KUNDU, S.D.; ROEHL, K.A.; EGGENER, S.E. y cols.: "Potency, continence and complications in 3,477 consecutive radical retropubic prostatectomies". J. Urol., 172: 2227, 2004.

66. QUINLAN, D.M.; EPSTEIN, J.I.; CARTER, B.S. y cols.: "Sexual function following radical prostatectomy: Influence of preservation of neurovascular bundles".J. Urol., 145: 998, 1991.

67. CATALONA, W.J.; BASLER, J.W.: "Return of erections and urinary continence following nerve sparing radical retropubic prostatectomy". J. Urol., 150: 905, 1993.

*68. DUBBELMAN, Y.D.; DOHLE, G.R.; SCHRODER, F.H.: "Sexual function before and after radical retropubic prostatectomy: A systematic review of prognostic indicators for a successful outcome". Eur. Urol., 50: 711, 2006.

69. MICHL, U.H.; FRIEDRICH, M.G.; GRAEFEN, M. y cols.: "Prediction of postoperative sexual function after nerve sparing radical retropubic prostatectomy". J. Urol., 176: 227, 2006.

70. MONTORSI, F.; BRIGANTI, A.; SALONIA, A. y cols.: "Current and future strategies for preventing and managing erectile dysfunction following radical prostatectomy". Eur. Urol., 45: 123, 2004.

*71. BURNETT, A.L.; LUE, T.F.: "Neuromodulatory therapy to improve erectile function recovery outcomes after pelvic surgery". J. Urol., 176: 882, 2006. 